\title{
Evaluation of Periarticular Soft Tissues in Patients With Juvenile Idiopathic Arthritis by Superb Microvascular Imaging and Shear Wave Elastography
}

\author{
Ömer Faruk ÜNAL@, Zuhal BAYRAMOĞLU ID, İbrahim ADALETLİ® \\ Department of Radiology, Istanbul University, Istanbul Medical Faculty, Istanbul, Turkey
}

\begin{abstract}
Objectives: This study aims to compare the vascularity and elasticity of periarticular soft tissues by superb microvascular imaging (SMI) and power Doppler (PD) ultrasound along with shear wave elastography (SWE) between children with juvenile idiopathic arthritis (JIA) and healthy children.

Patients and methods: This prospective single center study, conducted between March 2018 and May 2018, included 22 children with JIA (14 males, 8 females; mean age $11.3 \pm 5$ years; range, 5 to 17 years) and 24 healthy pediatric volunteers (12 males, 12 females; mean age $13 \pm 5.5$ years; range, 7 to 17 years). Quadriceps tendon (QT), patellar tendon (PT), infraarticular and supraarticular soft tissue elasticities were calculated via SWE. Supraarticular and infraarticular soft tissue vascularity index (VI) were evaluated via SMI and PD.

Results: No significant difference was found among the mean ages of the participants in study and control groups. Mean VI of both supraarticular $(8.15 \%)$ and infraarticular soft tissues $(7.9 \%)$ by SMI were significantly higher in study group compared to control group ( $2.88 \%$ vs. $2.57 \%$, respectively). Mean VI of both supraarticular (9.1\%) and infraarticular soft tissues (8.12\%) by PD were significantly higher in study group compared to control group ( $3.4 \%$ vs. $3.1 \%$, respectively). Highly significant good positive correlation was found between VI values obtained with PD and $S M I(r=0.9, p=0.001)$. There was a significant moderate positive correlation between the mean elasticity of the QT with VI of the supraarticular soft tissues by SMI ( $r=0.4$, $p=0.003)$. There was a significant moderate positive correlation of VI of infraarticular soft tissues by SMI with mean elasiticity of PT $(r=0.42, p=0.002)$. Conclusion: Vascularity index by SMI and PD could differentiate patients with JIA from healthy subjects. The SWE examination of tendons and soft tissues did not show any significant difference among patients with JIA and healthy subjects.

Keywords: Arthritis, elasticity, shear wave elastography, superb microvascular imaging, vascularity.
\end{abstract}

Juvenile idiopathic arthritis (JIA) is the most common chronic rheumatic disease that causes long-term disability in children. There are several types of JIA: polyarticular, oligoarticular and systemic onset which is accompanied with fever. ${ }^{1}$ JIA is diagnosed in children younger than 16 years of age, suffering from fever, pain, and limitation of motion lasting longer than six weeks after exclusion of other causes of arthritis. ${ }^{2}$ Imaging modalities for diagnosing JIA includes gray scale and Doppler ultrasound (US) along with magnetic resonance imaging (MRI). Gray scale US evaluation provides qualitative imaging findings as synovial thickening, presence of joint effusion and change in periarticular soft tissue echogenicity. ${ }^{3}$ Doppler US evaluation provides visualization of increased vascularization that has an important role in development or progression of inflammation. As a further diagnostic imaging modality, contrast enhanced joint MRI may be performed in patients with suspected acute arthritis. However, MRI would require sedation

Received: May 30, 2019 Accepted: August 25, 2019 Published online: February 07, 2020

Correspondence: Zuhal Bayramoğlu, MD. İstanbul Üniversitesi İstanbul Tıp Fakültesi Radyoloji Anabilim Dalı, 34093 Fatih, İstanbul, Türkiye. Tel: +90 544 - 8571278 e-mail: incezuhal@yahoo.com 
and contrast agent administration in the diagnosis of synovitis in children contrary to US that is a harmless, non-invasive, simple and initial imaging modality.

Recently, there are novel US based applications providing better visualization and quantification of microvascular network without using a contrast medium such as superb microvascular imaging (SMI). Quantification of viscoelastic tissue properties by US based modalities has been possible with shear wave elastography (SWE). SMI and SWE have been performed in differentiation of acute lymphadenitis from lymphoma, ${ }^{4}$ and in detection of vascularity changes in chronic autoimmune thyroiditis in children. ${ }^{5}$ In terms of musculoskeletal applications, SMI and SWE have been investigated in determination of effects of passive muscle stiffness on athletic performance. ${ }^{6}$ The previous studies concluded that vascularity could be quantificated by SMI with a novel parameter as vascularity index (VI) that has been valuable in detection of inflammatory changes. Although it is easy to detect joint effusion during evaluation of joint US in daily routine radiological practice, the final decision of synovitis due to arthritis requires experience. Difficulties in identifying synovitis may be due to the lack of numerical data for normal blood supply of synovium and periarticular soft tissues. A recently published study investigated the quantitative assessment of synovial vascularity by SMI compared to power Doppler (PD) US. ${ }^{6}$ Another recent study suggested the superiority of SMI compared to PD US without a significant interobserver difference. $^{7}$

Potential complications of JIA particularly in delayed diagnosis or undertreated cases have been reported to include joint contractures, muscle wasting, localized or generalized growth disturbances and impaired functioning. ${ }^{8}$ It would be of significant assistance to be able to predict the presence of active inflammation in terms of VI as percent (\%) by SMI and fibrosis-related complications based on quantitative data as kilopascals $(\mathrm{kPa})$ by SWE in the first joint ultrasonographic examination in the radiology unit. Therefore, in this study, we aimed to compare the vascularity and elasticity of periarticular soft tissues by SMI and PD US along with SWE between children with JIA and healthy children.

\section{PATIENTS AND METHODS}

This study was conducted at Istanbul University, Istanbul Medical Faculty between March 2018 and May 2018. Twenty-two children (14 males, 8 females; mean age $11.3 \pm 5$ years; range, 5 to 17 years) diagnosed with JIA and 24 healthy pediatric volunteers (12 males, 12 females; mean age $13 \pm 5.5$ years; range, 7 to 17 years) were examined with gray scale US for confirmation of knee joint arthritis in study group and normal findings of knee joints in control group. The selected subjects were evaluated quantitatively with PD, SMI and SWE in terms of infraarticular and supraarticular soft tissue vascularity including the synovium and fat pads of the knee joints in addition to quadriceps tendon (QT) and patellar tendon (PT) elasticity. Thirty-one knee joints of 22 JIA patients admitted to the pediatric rheumatology outpatient clinic with complaints of knee joint swelling or arthralgia and clinically diagnosed with knee joint arthritis were examined with SMI, PD and SWE within the same day of admission. The parameters for diagnosis of arthritis were joint swelling, tenderness, pain, restricted motion on physical examinations, and erythrocyte sedimentation rate and C-reactive protein by biochemical analysis. Exclusion criteria were being routine control patients diagnosed with JIA who were admitted to pediatric rheumatology department without an acute knee joint symptom, arthralgia, or fever as well as patients aged older than 18 years who were under treatment and asymptomatic or who had severe joint contractures. Healthy volunteers were selected among patients admitted to pediatric radiology department either for thyroid or neck US examination because of previously detected transient thyroid dysfunction or among adolescents who had no complaint of chronic fever, joint swelling, arthralgia and referred for hepatobiliary US check up. We evaluated bilateral joints of healthy volunteers by SMI, PD and SWE. The study protocol was approved by the Istanbul University, Istanbul Medical Faculty Ethics Committee (number: 2018/282). A written informed consent was obtained from parents of each participant. The study was conducted in accordance with the principles of the Declaration of Helsinki. 

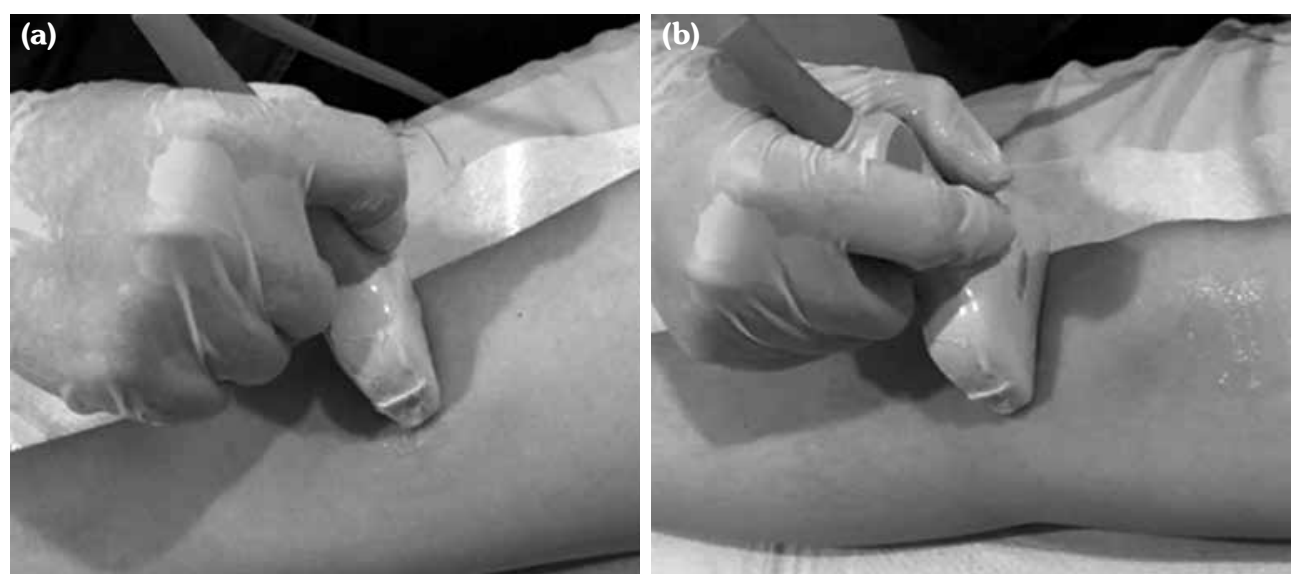

Figure 1. Evaluation of periarticular soft tissues with shear wave elastography, superb microvascular imaging and power Doppler. Transducer was placed just superior to patella perpendicular to long axis of knee joint demonstrating supraarticular recess of knee joint regarding supraarticular soft tissues (a), just inferior to patella demonstrating infraarticular recess of knee joint regarding infraarticular soft tissues (b).

All patients and healthy volunteers were screened with Canon Aplio 500 Platinum US device (Canon Medical Systems, Tokyo, Japan) equipped with SMI, PD and SWE functions. All participants were examined in supine position and their knees were in extension (Figures 1,2). Once the gray scale US examination confirmed the arthritis based on synovial thickening, joint effusion and periarticular echogenicity, then SMI and SWE were performed to the supraarticular and infraarticular soft tissues concerning the synovium and periarticular soft tissues. VI is a novel parameter determined for SMI and PD corresponding the ratio of colored pixels to the total pixels within the selected region of interest (ROI). VI was calculated automatically on colored SMI and PD images over the selected rectangular shaped ROI with edges measured $5 \times 15 \mathrm{~mm}$ (Figure 3). Mean VI values were calculated by averaging results of three different acquisitions of PD and SMI. The ROI was placed perpendicular to the long axis of the knee joint at the supraarticular and infraarticular locations including the synovium and periarticular soft tissues. On SWE evaluation, the QT and PT were demonstrated along with their long axis. Three different ROIs for SWE were performed from the tendon halves closer to the knee joints. Each ROI
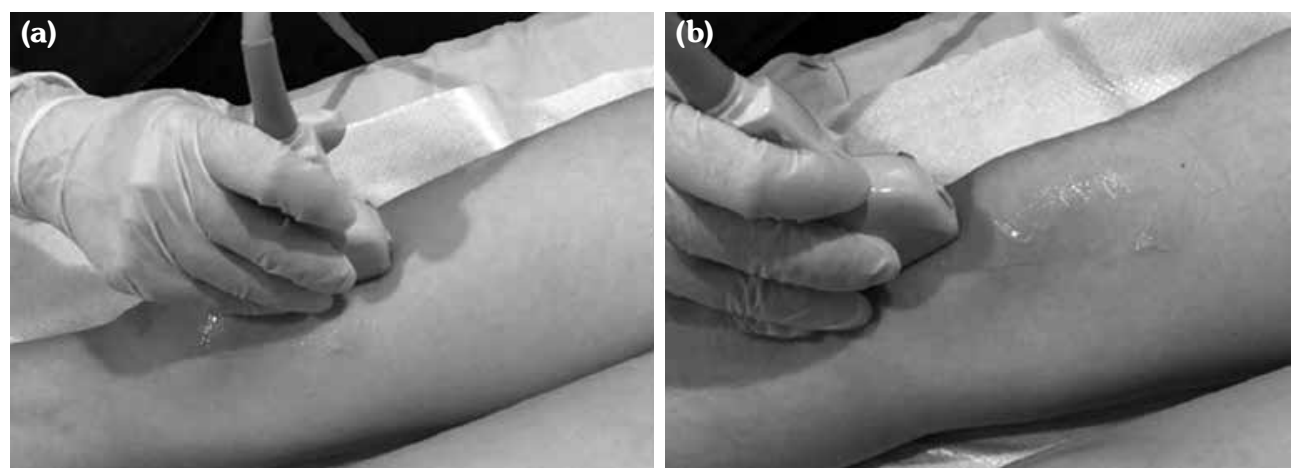

Figure 2. Evaluation of patellar and quadriceps tendons with shear wave elastography. Transducer was placed (a) just superior to patella parallel to long axis of knee joint demonstrating quadriceps tendon (b), just inferior to patella demonstrating patellar tendon. 

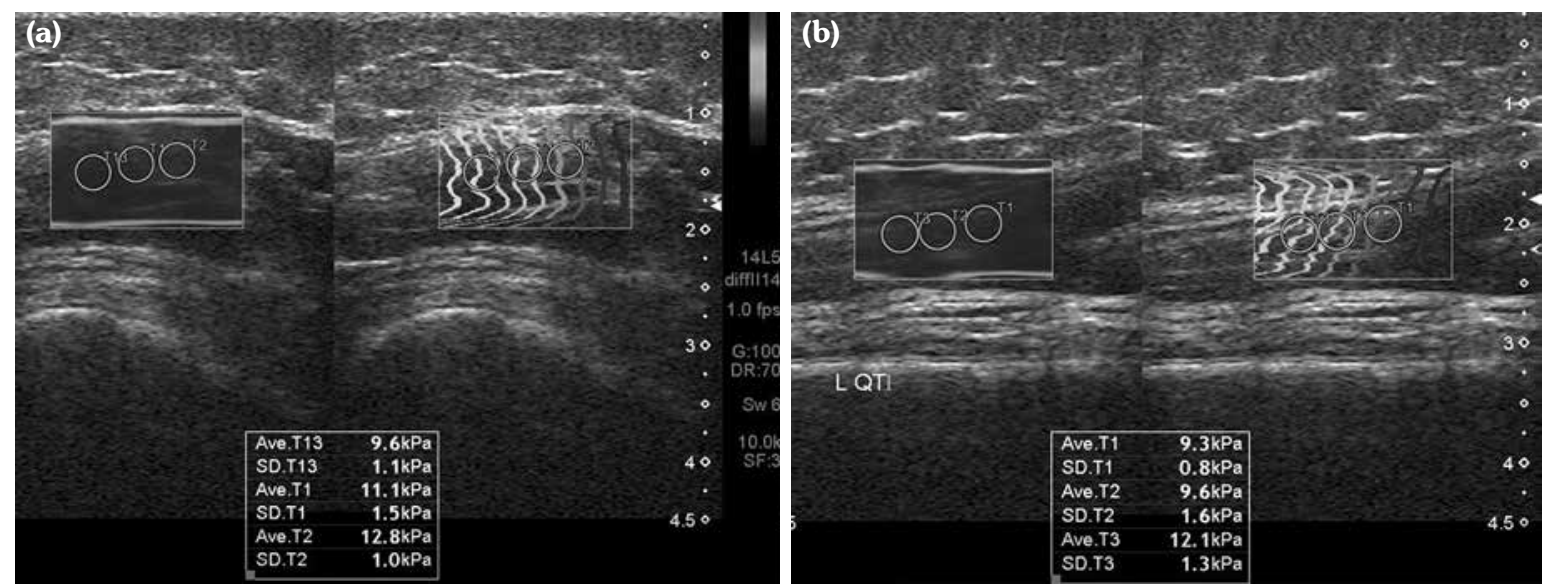

Figure 3. Evaluation of periarticular soft tissues with shear wave elastography. Shear wave elastography image (a) of supraarticular soft tissues by suprapatellar approach while transducer is perpendicular to long axis of knee joint, (b) shear wave elastography image of quadriceps tendon while transducer is parallel to long axis of knee joint.

was in circle shape and $3 \mathrm{~mm}$ in diameter. We depicted mean elasticity for PT, QT, supraarticular soft tissues (SAST) and infraarticular soft tissues (IAST) (Figures 4, 5).

\section{Statistical analysis}

Statistical analysis was performed using the IBM SSPS version 22.0 (IBM Corp., Armonk, NY, USA). The normal distribution of the data was evaluated by Kolmogorov-Smirnov test. Descriptive statistics of ages of the participants, VI for SMI and PD, and SWE were expressed with minimum, maximum, mean, and standard deviation. Student t-test was used to compare the VI via SMI and PD, and SWE values between the study and control groups. Pearson correlation analysis was used to evaluate the relationship between periarticular vascularity and elasticity parameters. Sensitivity, specificity and predictive values of VI and SWE along with optimal cut-off values for JIA related arthritis were calculated on receiver operating characteristic (ROC) curves.

\section{RESULTS}

No statistically significant difference was found between the mean ages of study and control subjects. Mean VI values in the study group obtained from SAST including synovium and suprapatellar fat pad were $8.2 \pm 3.4 \%$ for SMI and
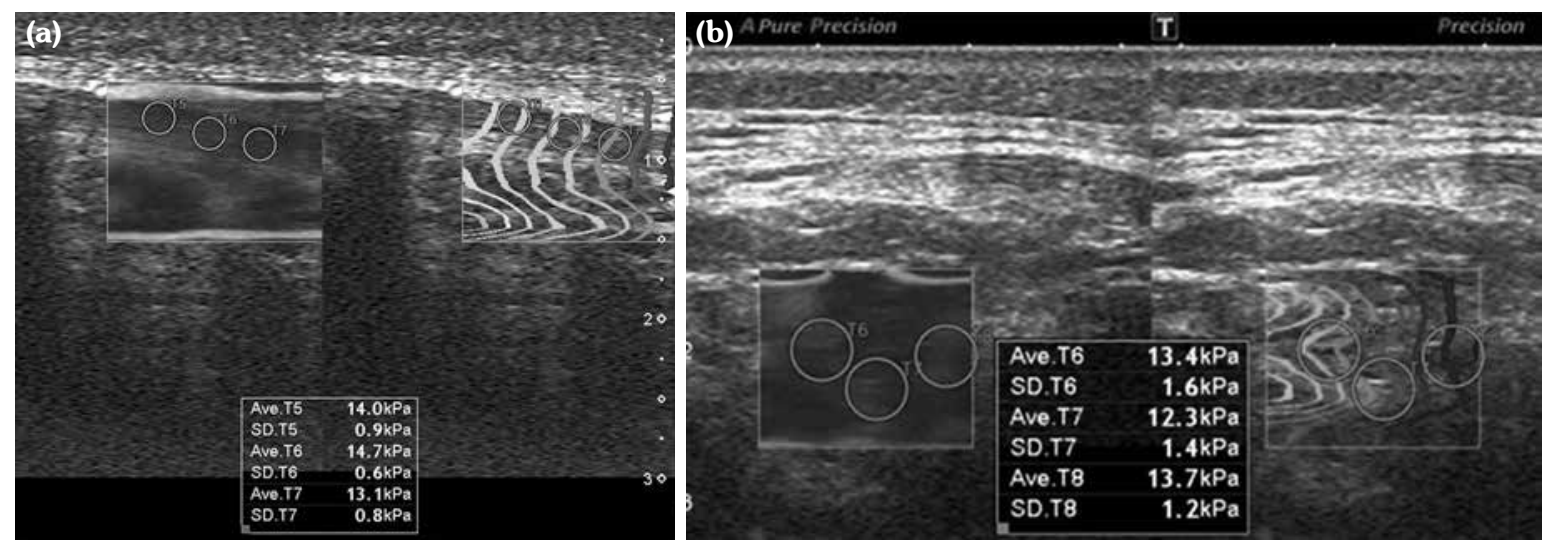

Figure 4. Evaluation of periarticular soft tissues with shear wave elastography. Shear wave elastography image (a) of patellar tendon while transducer is parallel to long axis of knee joint and shear wave elastography image (b) of infraarticular soft tissues by infrapatellar approach while transducer is perpendicular to long axis of knee joint. 

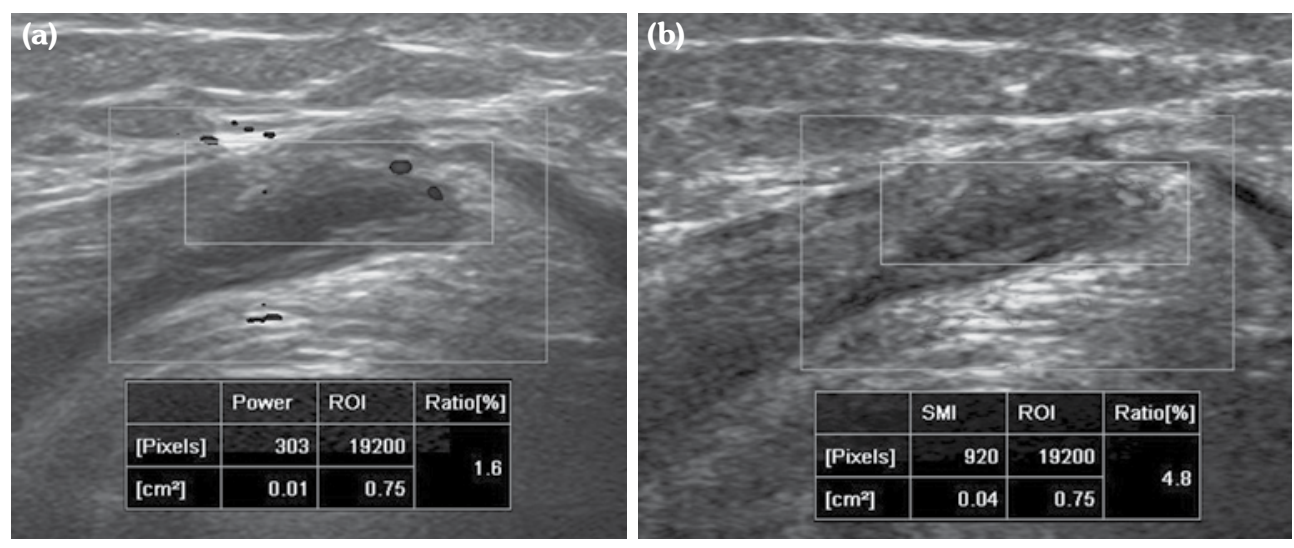

Figure 5. Evaluation of periarticular soft tissues with superb microvascular imaging and power Doppler. Evaluation of supraarticular soft tissues vascularity by percent (vascularity index) within region of interest $(5 \times 15 \mathrm{~mm})$. Vascularity index obtained via power Doppler (a) and vascularity index obtained by superb microvascular imaging (b) are given automatically.

$9.1 \pm 3.1 \%$ for PD. Those were found as $2.9 \pm 1.4 \%$ for SMI and $3.4 \pm 1.4 \%$ for $\mathrm{PD}$ in the control group. When the study and control groups were compared, there was a statistically significant difference between VI values determined by SMI $(p<0.001)$ and PD $(p<0.002)$, respectively. In the study group, mean VI values obtained from IAST including synovium and infrapatellar fat pad were $7.9 \pm 3.1 \%$ for SMI and $8.1 \pm 2.8 \%$ for PD. Those were found as $2.6 \pm 1.5 \%$ for SMI and $3.1 \pm 1.5 \%$ for PD in the control group. There was a statistically significant difference for VI values of IAST via SMI $(p<0.001)$ and PD $(p<0.003)$ among the study and control groups, respectively. A comparison of overall VI values obtained from both study and control group participants did not show any statistically significant difference among mean values ( $p>0.05)$.

The mean SWE values obtained from SAST including synovium and suprapatellar fat pad was $10.7 \pm 2.5 \mathrm{kPa}$ in study group and $9.9 \pm 2.3 \mathrm{kPa}$

Table 1. Descriptive statistics of age (years), patellar tendon elasticity, quadriceps tendon elasticity, supraarticular soft tissue elasticity, infraarticular soft tissue elasticity and vascularity index via superb microvascular imaging and power Doppler

\begin{tabular}{|c|c|c|c|c|c|}
\hline & \multicolumn{2}{|c|}{ Study group } & \multirow[b]{2}{*}{$p$} & \multicolumn{2}{|c|}{ Control group } \\
\hline & Mean \pm SD & Min-Max & & Mean \pm SD & Min-Max \\
\hline Age & $11.2 \pm 5$ & $5-18$ & 0.7 & $13 \pm 5.5$ & $7-17$ \\
\hline PT SWE & $19.7 \pm 6.9$ & $11-38$ & 0.16 & $16.5 \pm 4.8$ & $8.5-34$ \\
\hline QT SWE & $19 \pm 6.1$ & $10-34$ & 0.09 & $15.7 \pm 4.8$ & $9.4-26$ \\
\hline SAST SWE & $9.8 \pm 2.4$ & $5.2-16$ & 0.55 & $9.9 \pm 2.3$ & $4.5-16.2$ \\
\hline IAST SWE & $9.8 \pm 2.4$ & 4.9-14.6 & 0.65 & $9.4 \pm 2.3$ & 4.9-14.6 \\
\hline SAST VI SMI & $8.2 \pm 3.4$ & $2.4-17.5$ & 0.001 & $2.9 \pm 1.4$ & $1.6-6.8$ \\
\hline SAST VI PD & $9.1 \pm 3.1$ & $3.2-15$ & 0.002 & $3.4 \pm 1.4$ & $1.9-7.4$ \\
\hline IAST VI SMI & $7.9 \pm 3.1$ & $3.2-11.7$ & 0.001 & $2.6 \pm 1.5$ & $0.9-6.8$ \\
\hline IAST VI PD & $8.1 \pm 2.8$ & $3.9-15$ & 0.003 & $3.1 \pm 1.5$ & $1.6-8.4$ \\
\hline
\end{tabular}

SD: Standard deviation; Min: Minimum; Max: Maximum; PD: Power Doppler; SWE: Shear wave elastography; QT: Quadriceps tendon; SAST: Supraarticular soft tissues; IAST: Infraarticular soft tissues; VI: Vascularity index; SMI: Superb microvascular imaging; PT: Patellar tendon. 
Table 2. Correlation analysis of vascularity index among power Doppler and superb microvascular imaging, in addition to correlation among vascularity index and shear wave elasticity of periarticular soft tissues

\begin{tabular}{lcc}
\hline & $r$ & $p$ \\
\hline SAST VI (PD)-QT SWE & 0.9 & 0.001 \\
SAST VI (SMI)-QT SWE & 0.4 & 0.003 \\
IAST VI (PD)-PT SWE & 0.45 & 0.001 \\
IAST VI (SMI)-PT SWE & 0.42 & 0.002 \\
PD-SMI (VI) & 0.9 & 0.001 \\
\hline
\end{tabular}

SAST: Supraarticular soft tissues; VI: Vascularity index; PD: Power Doppler; QT: Quadriceps tendon; SWE: Shear wave elastography; SMI: Superb microvascular imaging; IAST: Infraarticular soft tissues; PT: Patellar tendon.

in the control group ( $p>0.05)$. The mean SWE values obtained from IAST including synovium and infraarticular fat pad was $9.8 \pm 2.4 \mathrm{kPa}$ in study group and $9.4 \pm 2.3 \mathrm{kPa}$ in the control group ( $p>0.05$ ). No significant difference was found among SWE values of periarticular soft tissues among study and control subjects. The mean SWE of PT was $19.7 \pm 6.9 \mathrm{kPa}$ in the study group and $16.5 \pm 4.8 \mathrm{kPa}$ in the control group. Although the mean QT SWE $(19 \pm 6.1 \mathrm{kPa})$ was higher in the study group than the control group $(15.7 \pm 4.8 \mathrm{kPa})$, the differences among the mean SWE values of QT and PT failed to reach statistically significant levels ( $p>0.05$ ) (Table 1).

There was a highly significant good positive correlation among VI values obtained via $\mathrm{PD}$ and SMI $(r=0.9, p=0.001)$. There was a moderate significant positive correlation between the mean
SWE values of QT with VI values obtained from SAST via SMI $(r=0.45, p=0.001)$ and also PD $(\mathrm{r}=0.4, \mathrm{p}=0.001)$ (Table 2). There was a moderate significant positive correlation between the mean SWE values of PT with VI values obtained from IAST via SMI $(r=0.41, p=0.003)$ and also PD $(\mathrm{r}=0.42, \mathrm{p}=0.002)$.

In order to distinguish JIA patients from healthy subjects, the optimal cut-off values, sensitivity, specificity, positive and negative predictive values and area under the curves were calculated on ROC curves. These values were 4.35\%, 95\%, $89 \%, 86 \%, 87 \%$, and $95 \%$ for VI via SMI obtained from SAST, respectively. These values were $4.15 \%, 91 \%, 89 \%, 87 \%, 85 \%$, and $96 \%$ for VI via SMI obtained from IAST, respectively. These values were 5.45\%, 91\%, 89\%, 85\%, 82\%, and $92 \%$ for VI via PD obtained from SAST, respectively. These values were 4.75\%, 95\%, $89 \%, 84 \%, 85 \%$, and $93 \%$ for VI via PD obtained from IAST, respectively. The area under the curve at 95\% safety level for SWE evaluation was 54\% for SAST, 55\% for IAST, 61\% for QT, and 62\% for PT, and they failed to achieve statistically significant diagnostic levels (Table 3).

\section{DISCUSSION}

Our results have revealed that in case of knee joint involvement with JIA, SMI may be used as a diagnostic tool and the VI values obtained by SMI and PD have demonstrated a considerable positive correlation. We have shown the applicability and distinctiveness of VI by SMI and PD for knee joint arthritis. This study has investigated both synovial and periarticular soft tissue vascularity along

Table 3. Diagnostic accuracy of vascularity index depicted on receiver operating characteristic curve analysis

\begin{tabular}{lcccccc}
\hline & $\begin{array}{c}\text { Cut-off } \\
(\%)\end{array}$ & $\begin{array}{c}\text { Sensitivity } \\
(\%)\end{array}$ & $\begin{array}{c}\text { Specificity } \\
(\%)\end{array}$ & $\begin{array}{c}\text { PPV } \\
(\%)\end{array}$ & $\begin{array}{c}\text { NPV } \\
(\%)\end{array}$ & $\begin{array}{c}\text { AUC } \\
(\%)\end{array}$ \\
\hline SAST VI (SMI) & 4.35 & 95 & 89 & 86 & 87 & 95 \\
IAST VI (SMI) & 4.15 & 91 & 89 & 87 & 85 & 96 \\
SAST VI (PD) & 5.45 & 91 & 89 & 85 & 82 & 92 \\
IAST VI (PD) & 4.75 & 95 & 89 & 84 & 85 & 93 \\
\hline
\end{tabular}

PPV: Positive predictive value; NPV: Negative predictive value; AUC: Area under the curve; SAST: Supraarticular soft tissues; VI: Vascularity index; SMI: Superb microvascular imaging; IAST: Infraarticular soft tissues; PD: Power Doppler. 
with viscoelastic tissue properties simultaneously. Similar with the recent study of Alis et al., ${ }^{7}$ we have found the VI parameter as a valuable and promising tool to detect synovial inflammation. From the technical perspective, Alis et al. ${ }^{7}$ have focused on the synovium alone by outlining the synovial borders while we have depicted a region from the recess including synovium. Therefore, a wider extrasynovial area would reduce the differences among the mean values of VI via PD and SMI. We appreciate the technique the authors have used; however, manually contouring the synovial borders would be time consuming and also might not be practical particularly in children and in routine examination. The technique we used has been unable to reveal the superiority of SMI to PD but has demonstrated clearly the distinctiveness of both SMI and PD for detection of synovial inflammation.

Chronic arthritis in childhood may result in sequelae as subluxation, contractures and deformities of involved joints. Although US-detected subclinical synovitis has not been determined as a predictor of flares in a recent study, ${ }^{8}$ increased rate of flare has been reported in patients in clinical remission but presenting positive Doppler findings. ${ }^{9}$ Thus early initial diagnosis and also detection of disease activity are crucial for achieving treatment response and reducing the sequelae. Since we have excluded patients with severe contractures, diagnostic accuracy of SWE for differentiation of JIA disease has failed to reach statistically significant levels. Increased soft tissue and tendon elasticity would be found in chronic arthritis particularly in cases with contractures. On the other hand, mean VI by SMI and PD and also their diagnostic accuracy would be found lower than the current results due to muscle atrophy and significant fibrosis when acute arthritis attack develops on a joint with chronic involvement. This study has shown the effects of acute arthritis on periarticular vascularity and elasticity. Further studies are required for investigating the effects of chronic arthritis on elasticity of periarticular soft tissues and tendons.

A recent US based acquisition protocol and scoring system have been developed for assessment of synovitis of knee joints in patients with JIA in terms of the suprapatellar, medial parapatellar and lateral parapatellar recesses via
B-mode US and PD US. ${ }^{10}$ We have evaluated quantitatively the vascularity of suprapatellar and infrapatellar recesses in integrity with adjacent tendons by SMI and PD. Reliability of the $B$ mode US and PD US has been discussed in several recent studies. One study ${ }^{11}$ concluded that more synovitis, tenosynovitis, cartilage damage and bone erosions could be detected via US compared to clinical examination and there has been good to excellent inter- and intraobserver reliability of US for the wrist and metacarpophalangeal joints. Furthermore, the superiority of SMI to PD in terms of diagnostic accuracy has been recently published without significant interobserver variability when measuring only synovial vascularity. ${ }^{7}$ The current study has demonstrated the comparison of the knee joints among acute JIA patients and healthy children in terms of visualization of microvascularization of soft tissues around knee joint and also quantification of the microvascular flow. As far as we know, objective and standard quantification of periarticular and synovial vascularity has been demonstrated only by VI either with PD or SMI in terms of Doppler US techniques. ${ }^{7}$ Scoring systems reveal disease activity with semiquantitative demonstration of vascularity by vascular codes.

Normative standards of knee joints of healthy children regarding synovial membrane thickness $(<1.8 \mathrm{~mm})$ and mean overall diameter of largest effusion pocket $(2.8 \mathrm{~mm})$ have been evaluated on contrast enhanced MRI. ${ }^{12}$ Quantification of periarticular evaluation in children has been investigated commonly and widely by MRI either with diffusion weighted or contrast enhanced sequences. Pharmacokinetic modeling of the dynamic contrast enhanced MRI have been also used in detection of early synovitis by calculating quantitative parameters. ${ }^{13}$ As an alternative to contrast administration, diffusion weighted MRI for evaluation of synovitis has been reported as a safer and more patient friendly imaging technique. ${ }^{14,15}$ Elevated acute phase reactants would be encountered in extended oligoarticular JIA patients. However, acute phase reactants would be within the normal range in some cases with acute arthritis and supporting radiological findings may be required. ${ }^{1}$ Although MRI is accepted as a gold standard modality for arthritis; a practical, easy, fast and radiation free method 
without need of sedation would be reasonably the first choice imaging modality. Therefore, further US based applications with standard protocols and quantifications seem to be promising.

Superb microvascular imaging is a novel Doppler imaging technique that may demonstrate low velocity flows in the microvascular network by using higher frame rates and lower pulse repetition frequency than $\mathrm{PD}$ without the need to use a contrast medium. Along with better visualization of fine vessels, SMI algorithm provides a novel quantification as VI corresponding the blood flow per tissue within the selected region by calculating the ratio of color pixels to the total pixels. Both clear visualization and also quantification of the microvascular signals would provide advantages particularly in inflammatory disorders. In order to eliminate contrast administration and other disadvantages of MRI particularly in children with suspected arthritis, microvascular imaging techniques such as SMI may be performed to demonstrate the initial attack of the arthritis and also disease activity.

There have been recent studies investigating the role of SMI for evaluation of active synovitis compared to $\mathrm{PD},{ }^{16,17}$ in evaluation of hand joint lesions in adults with rheumatoid arthritis, ${ }^{18}$ in terms of lateral epicondylosis diagnosis ${ }^{19}$ and synovial inflammation in rheumatic diseases compared to PD. ${ }^{20}$ Recent studies have demonstrated the superiority of SMI to PD and color Doppler., ${ }^{7,16}$ In the current study, we have revealed a highly significant positive correlation of VI obtained by SMI and PD but not their superiority to one another. Furthermore, to our knowledge, this is the first study in the literature investigating the applicability of the quantitative evaluation of SMI in JIA patients during knee joint US examination along with possible changes in periarticular soft tissue elasticity via SWE. Based on our results, VI values obtained via PD and SMI have been found to be significantly and positively correlated. Therefore, VI via PD may be reliably used alone in the decision making of acute arthritis. We have defined the range of cut-off values for VI between $4.15 \%$ and $5.45 \%$. With the help of quantitative evaluation, both the initial diagnosis of acute arthritis and also treatment response to different local and systemic treatments may be observed objectively during the follow-up of patients. During follow- up, it is necessary to evaluate the patients with standard protocols such as placing the ROI at the suprapatellar recess parallel to the long axis of the QT.

Differentiation of the etiology of synovitis either as rheumatological or infectious causes has been a common diagnostic dilemma in radiology practice unless the effusion has been aspirated. Effusions with heterogeneous contents due to thickened synovium, cartilage damage or bony erosions could be mostly due to inflammatory arthritis rather than transient effusion or septic arthritis. Thickened synovium along with increased vascularity suggest JIA involvement or flare in a child knee rather than septic arthritis. We could demonstrate increased vascularity in JIA but not distinctive values from septic arthritis. By providing quantitative values with SMI, VI may be investigated in the differentiation of septic from inflammatory arthritis in future studies.

Shear wave elastography is also called dynamic elastography and uses "acoustic radiation force sequence to generate shear waves propagating perpendicularly to the US beam" 21 causing tissue displacement. Changes in the shear wave velocities are associated with viscoelastic tissue properties and calculated based on the shear modulus that can be calculated by multiplying tissue density and square of the shear speed. ${ }^{21}$ Shear waves propagate faster in stiffer tissues. The viscoelastic properties of tendons may be changed due to investigated tendon parts, duration of the inflammation or presence of joint contractures. The combined evaluation of periarticular soft tissues with color Doppler and SWE has been found to increase the diagnostic accuracy in terms of determining acute phase of gout from intercritical phase for the first metatarsophalangeal joints. ${ }^{22}$ Although we have not found significant differences among mean SWE values, they were higher in the study group. Since normative tendon elasticity values have not been determined for children, serial measurements during follow-up may reveal chronicity and also support disease activity when combined with SMI.

Elasticity changes in tendons in case of arthritis have been evaluated in Achilles tendons ${ }^{23}$ revealing softening of the distal third of the tendon due to enthesopathy. Effects of arthritis on biomechanical properties of PT have been 
investigated ${ }^{24}$ and found to be lower in patients with arthritis compared to healthy controls. PT elasticity has been evaluated in a few studies ${ }^{25}$ in athletes revealing higher shear elastic modulus in painful tendons. Increased stiffness of tendons has been demonstrated in contracted tendons and decreased tendon elasticity has been found in patients with tendinopathy. ${ }^{21}$ The association of strain ratio and biomechanical indicators has been revealed in a recent study investigating Achilles tendon elasticity in patients with cerebral palsy by real time US elastography. ${ }^{26}$ Therefore, the tendon parts, the symptoms and status of the range of motion may affect the results of SWE investigation. Further studies including more participants, tendon parts and types are needed to clarify the effects of maturation, normal aging and also tendinopathies in children. Moreover, the effects of acute knee arthritis on PT and QT elasticity have yet to be demonstrated. With this study, we have revealed SWE changes in PT and QT in pediatric JIA patients presented with acute arthritis as preliminary results. The different results obtained in our study may have been caused by the included pediatric population, the duration of the disease activity, the medication status or the dynamic status of musculoskeletal evaluation although the patients were all in the supine position without knee flexion. Since development of fibrosis and contracture requires a long disease activity period, ${ }^{27}$ the differences in elasticity may be occult in early childhood and within the initial activity periods. The negligible increase of SWE values in tendons may be further investigated in studies including more participants and wide range of follow-up period including both acute arthritis and chronic follow-up patients.

There are several limitations of our study. Although arthritis was confirmed with US examination, and clinical and laboratory findings; the medication status and duration of the disease were not subdivided and compared among each other because of the limited number of cases. We also did not include patients with effusion but included those in clinical remission as a comparative study subgroup to reveal whether the VI could differentiate flares from chronic effusion with residual synovial hypertrophy. Furthermore, the PD, SMI and SWE images were obtained by the same operator and therefore we were unable to perform interobserver variability.
Lastly, we did not evaluate parapatellar spaces, which were suggested for examination in recent scoring systems, ${ }^{7}$ because it is technically difficult to place the transducer and obtain a wide ROI consisting of soft tissues to investigate vascularity. In addition, severe artifacts prevent optimal measurement of elasticity around parapatellar spaces due to bony structures.

In conclusion, there was no significant difference in elasticity between the PT and QT, and periarticular soft tissues of JIA patients compared to control subjects. However, the VI values obtained with SMI and PD were significantly higher in patients with JIA compared to healthy subjects. Combined studies with SMI and SWE would be promising for detection of initial diagnosis and activity of JIA.

\section{Declaration of conflicting interests}

The authors declared no conflicts of interest with respect to the authorship and/or publication of this article.

\section{Funding}

The authors received no financial support for the research and/or authorship of this article.

\section{REFERENCES}

1. Barut K, Adrovic A, Şahin S, Kasapçopur Ö. Juvenile idiopathic arthritis. Balkan Med J 2017;34:90-101.

2. Weiss JE, Ilowite NT. Juvenile idiopathic arthritis. Pediatr Clin North Am 2005;52:413-42

3. Kofler J, Altenbrunner-Martinek B. Ultrasonographic findings of disorders of the tarsal region in 97 cattle--arthritis, bursitis, tenosynovitis, periarticular abscess and vein thrombosis. Berl Munch Tierarztl Wochenschr 2008;121:145-58.

4. Bayramoglu Z, Caliskan E, Karakas Z, Karaman S, Tugcu D, Somer A, et al. Diagnostic performances of superb microvascular imaging, shear wave elastography and shape index in pediatric lymph nodes categorization: a comparative study. $\mathrm{Br}$ J Radiol 2018;91:20180129.

5. Bayramoglu Z, Kandemirli SG, Caliskan E, Yilmaz $\mathrm{R}$, Kardelen AD, Poyrazoglu S, et al. Assessment of paediatric Hashimoto's thyroiditis using superb microvascular imaging. Clin Radiol 2018;73:1059. e9-1059.e15.

6. Akkoc O, Caliskan E, Bayramoglu Z. Effects of passive muscle stiffness measured by Shear Wave Elastography, muscle thickness, and body mass index on athletic performance in adolescent female basketball players. Med Ultrason 2018;20:170-6. 
7. Alis D, Erol BC, Akbas S, Barut K, Kasapcopur O, Adaletli I. Superb microvascular imaging compared with power doppler ultrasound in assessing synovitis of the knee in juvenile idiopathic arthritis: A preliminary study. J Ultrasound Med 2019. [Epub ahead of print]

8. Nieto-González JC, Rodríguez A, Gámir-Gámir ML, Boteanu A, López-Robledillo JC, Garulo DC, et al. Can ultrasound-detected subclinical synovitis be an indicator of flare recurrence in juvenile idiopathic arthritis remission patients on tapered TNFi? Clin Exp Rheumatol 2019;37:705-12.

9. Spârchez M, Fodor D. What's new in musculoskeletal ultrasound in pediatric rheumatology? Med Ultrason 2018;20:371-8.

10. Ting TV, Vega-Fernandez P, Oberle EJ, De Ranieri D, Bukulmez H, Lin C, et al. Novel Ultrasound Image Acquisition Protocol and Scoring System for the Pediatric Knee. Arthritis Care Res (Hoboken) 2019;71:977-85.

11. Ventura-Ríos L, Faugier E, Barzola L, De la CruzBecerra LB, Sánchez-Bringas G, García AR, et al. Reliability of ultrasonography to detect inflammatory lesions and structural damage in juvenile idiopathic arthritis. Pediatr Rheumatol Online J 2018;16:58.

12. Hemke R, van den Berg JM, Nusman CM, van Gulik EC, Barendregt AM, Schonenberg-Meinema D, et al. Contrast-enhanced MRI findings of the knee in healthy children; establishing normal values. Eur Radiol 2018;28:1167-74.

13. Maijer KI, van der Leij C, de Hair MJ, Tas SW, Maas M, Gerlag DM, et al. Dynamic contrast-enhanced magnetic resonance imaging using pharmacokinetic modeling: Initial experience in patients with early arthritis. Arthritis Rheumatol 2016;68:587-96.

14. Barendregt AM, van Gulik EC, Lavini C, Nusman $\mathrm{CM}$, van den Berg JM, Schonenberg-Meinema D, Diffusion-weighted imaging for assessment of synovial inflammation in juvenile idiopathic arthritis: a promising imaging biomarker as an alternative to gadolinium-based contrast agents. Eur Radiol 2017;27:4889-99.

15. Hilbert F, Holl-Wieden A, Sauer A, Köstler H, Neubauer H. Intravoxel incoherent motion magnetic resonance imaging of the knee joint in children with juvenile idiopathic arthritis. Pediatr Radiol 2017;47:681-90.

16. Lee GY, Kim S, Choi ST, Song JS. The superb microvascular imaging is more sensitive than conventional power Doppler imaging in detection of active synovitis in patients with rheumatoid arthritis.
Clin Rheumatol 2019;38:2613-20.

17. Orlandi D, Gitto S, Perugin Bernardi S, Corazza A, De Flaviis L, Silvestri E, et al. Advanced Power Doppler Technique Increases Synovial Vascularity Detection in Patients with Rheumatoid Arthritis. Ultrasound Med Biol 2017;43:1880-7.

18. Yu X, Li Z, Ren M, Xi J, Wu J, Ji Y. Superb microvascular imaging (SMI) for evaluating hand joint lesions in patients with rheumatoid arthritis in clinical remission. Rheumatol Int 2018;38:1885-90.

19. Arslan S, Karahan AY, Oncu F, Bakdik S, Durmaz MS, Tolu I. Diagnostic performance of superb microvascular imaging and other sonographic modalities in the assessment of lateral epicondylosis. J Ultrasound Med 2018;37:585-93.

20. Yokota K, Tsuzuki Wada T, Akiyama Y, Mimura T. Detection of synovial inflammation in rheumatic diseases using superb microvascular imaging: Comparison with conventional power Doppler imaging. Mod Rheumatol 2018;28:327-33.

21. Taljanovic MS, Gimber LH, Becker GW, Latt LD, Klauser AS, Melville DM, et al. Shear-wave elastography: Basic physics and musculoskeletal applications. Radiographics 2017;37:855-70.

22. Wang Q, Guo LH, Li XL, Zhao CK, Li MX, Wang $\mathrm{L}$, et al. Differentiating the acute phase of gout from the intercritical phase with ultrasound and quantitative shear wave elastography. Eur Radiol 2018;28:5316-27.

23. Turan A, Tufan A, Mercan R, Teber MA, Tezcan ME, Bitik B, et al. Real-time sonoelastography of Achilles tendon in patients with ankylosing spondylitis. Skeletal Radiol. 2013;42:1113-8.

24. Matschke V1, Jones JG, Lemmey AB, Maddison PJ, Thom JM. Patellar tendon properties and lower limb function in rheumatoid arthritis and ankylosing spondylitis versus healthy controls: a cross-sectional study. ScientificWorldJournal 2013;2013:514743.

25. Zhang ZJ, Ng GY, Lee WC, Fu SN. Changes in morphological and elastic properties of patellar tendon in athletes with unilateral patellar tendinopathy and their relationships with pain and functional disability. PLoS One 2014;9:e108337.

26. Öztürk M, Sayinbatur B. Real-time ultrasound elastography of the Achilles tendon in patients with cerebral palsy: is there a correlation between strain ratio and biomechanical indicators? J Med Ultrason (2001) 2018;45:143-8.

27. Challenge for timely diagnosis of juvenile idiopathic arthritis in children. Paediatr Child Health 2008;13:192. 